\title{
Cervicofacial infection of dental origin presenting to maxillofacial surgery units in the United Kingdom: a national audit
}

- There is a reported increase in cervicofacial infections of dental origin presenting to oral and maxillofacial surgery units.

- Lack of access to primary care dental services was implicated as the root cause.

- Two thirds of patients treated by dental primary care services received inadequate treatment.

- Many patients with cervicofacial infection of dental origin present to primary care medical services.

\author{
L. M. Carter ${ }^{1}$ and S. Layton ${ }^{2}$
}

VERIFIABLE CPD PAPER

\begin{abstract}
Introduction Local studies have shown an increase in cervicofacial infections of dental origin presenting to oral and maxillofacial surgery units in the UK. A lack of access to National Health Service (NHS) primary care dental services has been implicated as a root cause. Study design Cross-sectional national audit. Method Oral and maxillofacial surgery units in the UK were asked to report details of severe cervicofacial infection of dental origin presenting in October and November 2006. Data were collected regarding: patient demographics, referral source, management in primary care, management by maxillofacial surgery, and outcome. Results Two hundred and sixty-six episodes of cervicofacial infection of dental origin were reported during the audit period. At the time of presentation, 56.4\% of patients were registered with a general dental practitioner (GDP). One hundred and forty (52.6\%) patients sought treatment from primary care dental services for their episode of cervicofacial infection and only 20 patients were unsuccessful in obtaining treatment. Forty-seven percent of patients did not seek treatment from primary care dental services. Fifty percent of patients were referred by accident and emergency. Sixty-six percent of patients were prescribed oral antibiotics without operative intervention by primary dental care services. Eighty-one percent of patients required hospital admission and $46 \%$ of patients required a surgical procedure under general anaesthesia. Eighty-nine percent of patients made a full recovery and 3\% recovered with complications. There were no deaths reported during the audit period. Discussion This audit provides a benchmark from which future comparisons can be made and by design cannot prove an increase in the presentation of cervicofacial infection of dental origin. Lack of access to NHS primary care dental services may be less significant than originally thought. A significant proportion of patients preferentially present to primary care medical services rather than dental services. Two thirds of patients treated by primary care dental services received oral antibiotics only which represents an inadequate level of treatment for odontogenic infection.
\end{abstract}

\section{INTRODUCTION}

Local studies have shown an increase in cervicofacial infections of dental origin presenting to oral and maxillofacial surgery units in the United Kingdom. ${ }^{1,2}$ The total number of hospital admissions and bed days as a result of drainage of a dental abscess in the UK has almost doubled between 1998-9 and 2005-6. ${ }^{3}$ Severity of presentation has also increased..$^{2,4-7}$ A lack of access to National Health

'British Association of Oral and Maxillofacial Surgeons Clinical Effectiveness Sub-Committee and Leeds Dental Institute, Oral and Maxillofacial Surgery, Clarendon Way, Leeds, LS2 9LU; ${ }^{2}$ British Association of Oral and Maxillofacial Surgeons Clinical Effectiveness Sub-Committee, Lincoln, LN2 50Y

*Correspondence to: Mr Lachlan Carte

Email: lachlan_carter@hotmail.com

\section{Refereed Paper}

Accepted 11 July 2008

DOI: $10.1038 /$ sj.bdj.2009.4

${ }^{\circ}$ British Dental Journal 2009; 206: 73-78
Service (NHS) primary care dental services following changes in service provision and remuneration of dentists over the past ten years has been implicated as a root cause, particularly in areas of high treatment need. ${ }^{3-8}$

As a consequence of these worrying trends the clinical effectiveness sub-committee of the British Association of Oral and Maxillofacial Surgeons (BAOMS) carried out a national audit of cervicofacial infection of dental origin presenting to oral and maxillofacial surgery units in the UK.

\section{METHOD}

Oral and maxillofacial surgery units in the UK were asked to report details of severe cervicofacial infection of dental origin presenting in October and November 2006. Data were collected using a standard proforma (Fig. 1). Data were collected regarding: patient demographics, referral source, management in primary care, management by maxillofacial surgery, and outcome. Socioeconomic class analysis was based on the Registrar General's classification of occupations (2000). For the purpose of this audit, severe cervicofacial infection was defined as 'Infection requiring more than just simple dental extraction/opening of tooth under local anaesthesia - ie could not be done by average GDP'.

\section{RESULTS}

Two hundred and sixty-six episodes of cervicofacial infection of dental origin were reported during the audit period. A normal distribution of age was evident and the mean patient age was 34.8 years (SD 15.6 years, range 1 to 83 years), with 155 male and 107 female patients 


\section{BAOMS Benchmark Study 2006-7: Cervicofacial infections presenting to OMFS}

PLEASE - Record all severe infections of dental origin

Record only patients requiring specialist intervention because of severity of problem

Definition of specialist intervention:

Any patient past the stage where they could be managed by a primary care dentist, including needing GA

HOSPITAL/UNIT NAME

\section{PATIENT DETAILS}

\begin{tabular}{|c|c|c|c|}
\hline Age & Sex $M / F$ & Occupation & \\
\hline & & e tick as appropriate) & \\
\hline Regular dentist (NHS) & $\square$ & General medical practitioner & \\
\hline Regular dentist (private) & $\square$ & Accident and emergency & \\
\hline Emergency dental service & $\square$ & Another hospital speciality & \\
\hline Dental access centre & $\square$ & Other (please state) & \\
\hline
\end{tabular}

\section{TREATMENT HISTORY}

\section{Is the patient registered with a general dental practitioner (GDP)?}

If No had the patient attempted to register with a GDP prior to this episode?

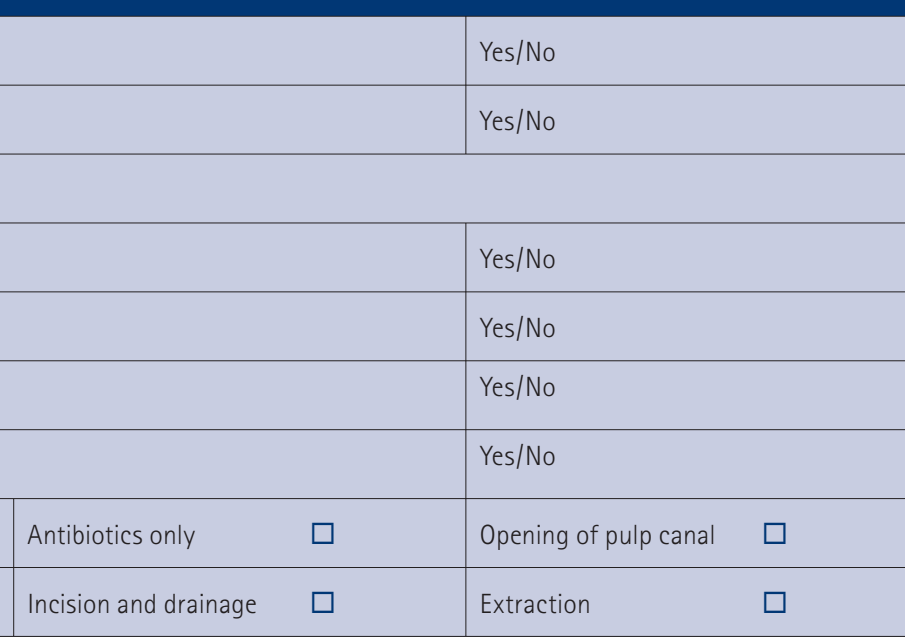

If unable to register was this because of:

\begin{tabular}{|c|c|c|c|c|}
\hline \multicolumn{3}{|l|}{ Lack of available NHS dentist in the area } & \multicolumn{2}{|l|}{ Yes/No } \\
\hline \multicolumn{3}{|l|}{ Unwilling to register with private dentist } & \multicolumn{2}{|l|}{ Yes/No } \\
\hline \multicolumn{3}{|l|}{ Did the patient seek treatment with dental services for this episode? } & \multicolumn{2}{|l|}{ Yes/No } \\
\hline \multicolumn{3}{|l|}{ Was the patient treated by dental services prior to presentation? } & \multicolumn{2}{|l|}{ Yes/No } \\
\hline \multirow[t]{2}{*}{ If Yes, what treatment was provided? (please tick as appropriate) } & Antibiotics only & $\square$ & Opening of pulp canal & $\square$ \\
\hline & Incision and drainage & $\square$ & Extraction & $\square$ \\
\hline
\end{tabular}

Management of patient by OMFS (please tick all that apply)

\section{Inpatient:}

IV antibiotics

Aspiration of pus

Intra oral incision and drainage LA

Intra oral incision and drainage GA

Extraction of teeth LA

Extraction of teeth $G A$

Extra oral incision and drainage LA

\begin{tabular}{|l|}
\hline Length of stay (nights): \\
\hline Extra oral incision and drainage GA \\
\hline Surgical airway \\
\hline Admission to ITU/ICU \\
\hline Surgical debridement of necrotic tissue \\
\hline Skin grafting \\
\hline Other (please state)
\end{tabular}

Length of stay (nights):

\section{$\square$}

$\square$

$\square$

$\square$

$\square$

$\square$

$\square$

Outcome (please tick as appropriate)

$\begin{array}{ll}\text { Full recovery } & \square \\ \text { Recovery with complications } & \square \\ \text { Death } & \square\end{array}$

Thank you for your cooperation

STUDY PERIOD: OCTOBER AND NOVEMBER 2006

Please return forms when completed to:

Stephen Layton, Maxillofacial Unit, County Hospital Lincoln, Lincoln LN2 50Y 
recorded and gender not recorded in four cases. Distribution of socioeconomic class is shown in Figure 2; the majority of patients were socioeconomic class III (non-manual) or V. The geographic distribution of the number of reported cases identified by postcode is shown in Figure 3.

At the time of presentation, 56.4\% of patients were registered with a general dental practitioner (GDP), but of those not registered $67.6 \%$ had not previously attempted to register with a GDP. The reasons for non-registration were poorly recorded but 48 patients reported a lack of GDPs in their area and 39 patients reported that they were unwilling to register with a private GDP.

Treatment provision by primary care dental services is shown in Table 1 and Figure 4. One hundred and forty (52.6\%) patients sought treatment from primary care dental services for their episode of cervicofacial infection. One hundred and twenty patients were treated by primary care dental services meaning only 20 patients who sought treatment were unsuccessful in obtaining treatment.

Figure 4 shows that $66 \%$ of patients were prescribed oral antibiotics only and less than $40 \%$ of patients had any form of operative dental treatment (incision and drainage, opening of pulp canal or extraction) by primary dental care services.

The source of referral to oral and maxillofacial surgery is shown in Table 2. Fifty percent of patients were referred by accident and emergency with only $25 \%$ referred by primary care dental services. Eighty-one percent of patients required hospital admission by oral and maxillofacial surgery with a median hospital stay of two nights (interquartile range 2 to 3 nights). The treatment provided by oral and maxillofacial surgery is shown in Figure 5. Forty-six percent of patients required general anaesthesia for dental extraction and/or intra-oral incision and abscess drainage. Eleven percent required general anaesthesia for extra-oral incision and abscess drainage and/or exploration of tissue spaces. Eighty-nine percent of patients made a full recovery and 3\% recovered with complications. There were no deaths reported during the audit period.

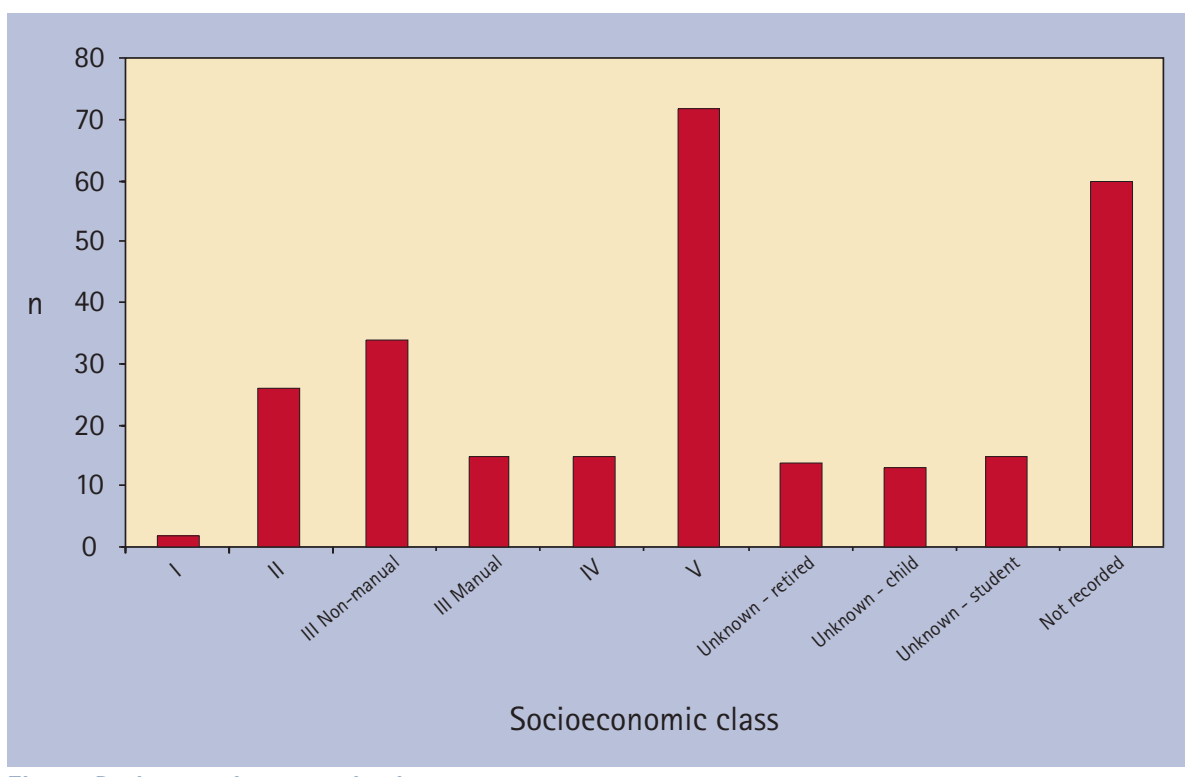

Fig. 2 Patient socioeconomic class

\begin{tabular}{|l|l|l|}
\hline Table 1 Treatment sought and provided by primary care dental services \\
\hline Treatment provision by primary care dental services & $\mathrm{n}$ & $\%$ \\
\hline Sought treatment & 140 & 52.6 \\
\hline Did not seek treatment & 125 & 47.0 \\
\hline Not recorded & 1 & 0.4 \\
\hline & $\mathrm{n}$ & $\%$ \\
\hline Treatment provided & 120 & 45.1 \\
\hline Treatment not provided & 145 & 54.5 \\
\hline Not recorded & 1 & 0.4 \\
\hline Sought treatment but unable to obtain & 20 & 7.5 \\
\hline
\end{tabular}

\begin{tabular}{|l|l|l|}
\hline Table 2 Patient referral source & $n$ & $\%$ \\
\hline Referral source & 134 & 50 \\
\hline ACtE & 41 & 15 \\
\hline GDP NHS & 25 & 9 \\
\hline GMP & 19 & 7 \\
\hline Another hospital specialty & 16 & 6 \\
\hline Emergency dental service & 10 & 4 \\
\hline Dental access centre & 4 & 2 \\
\hline GDP private & 2 & 1 \\
\hline NHS direct & 2 & 1 \\
\hline Self referral & 2 & 1 \\
\hline Other & 11 & 4 \\
\hline Not recorded & & \\
\hline
\end{tabular}

\section{DISCUSSION}

This audit provides a benchmark from which future comparisons can be made and by its cross-sectional design cannot prove an increase in the presentation of cervicofacial infection of dental origin. Not all oral and maxillofacial surgery units replied therefore the absence 
of data may represent non-compliance rather than a true absence of data. Also this audit is likely to under-estimate the true number of cases of cervicofacial infection of dental origin and therefore generalisations to all areas of the UK must be made with caution.

Changes in remuneration of dentists over the last decade has been reported to have caused a reduction in the proportion of NHS treatment carried out by general dentists. ${ }^{3}$ The number of NHS general dental practitioners decreased after a further change in dentists' remuneration by introduction of the new contract in April 2006. The NHS information centre reported a reduction of 1,649 (8\%) in NHS dental practitioners after introduction of the new contract in $2006 .{ }^{9}$ However, lack of access to primary care dental services may not be as important as originally thought. This study showed that of the $52.6 \%$ of patients who sought treatment from primary care dental services only 20 of these patients were unable to obtain treatment.

Interestingly $47 \%$ of patients did not seek treatment from primary care dental services and presented to accident and emergency or their general medical practitioner which is consistent with previous studies. ${ }^{1}$ Reasons for this were poorly recorded although some patients reported a lack of NHS general dental practitioners in their area and some patients were unwilling to register with a private dental practitioner. The cost of dental treatment may therefore be a reason for presentation to their general medical practitioner or accident and emergency and may be reflected in the fact that the majority of patients were from socioeconomic class V. There was a further peak of patients from socioeconomic class III (non-manual) who may be unable to access private dental services due to cost and cannot access exempt care because their earnings are too high. In addition, the more immediate availability of the patients' general medical practitioner or accident and emergency may be another reason for preferential presentation. Although not recorded in this audit dental phobia or previous bad experiences may also be reasons not to present to a GDP. Of the total number of patients,

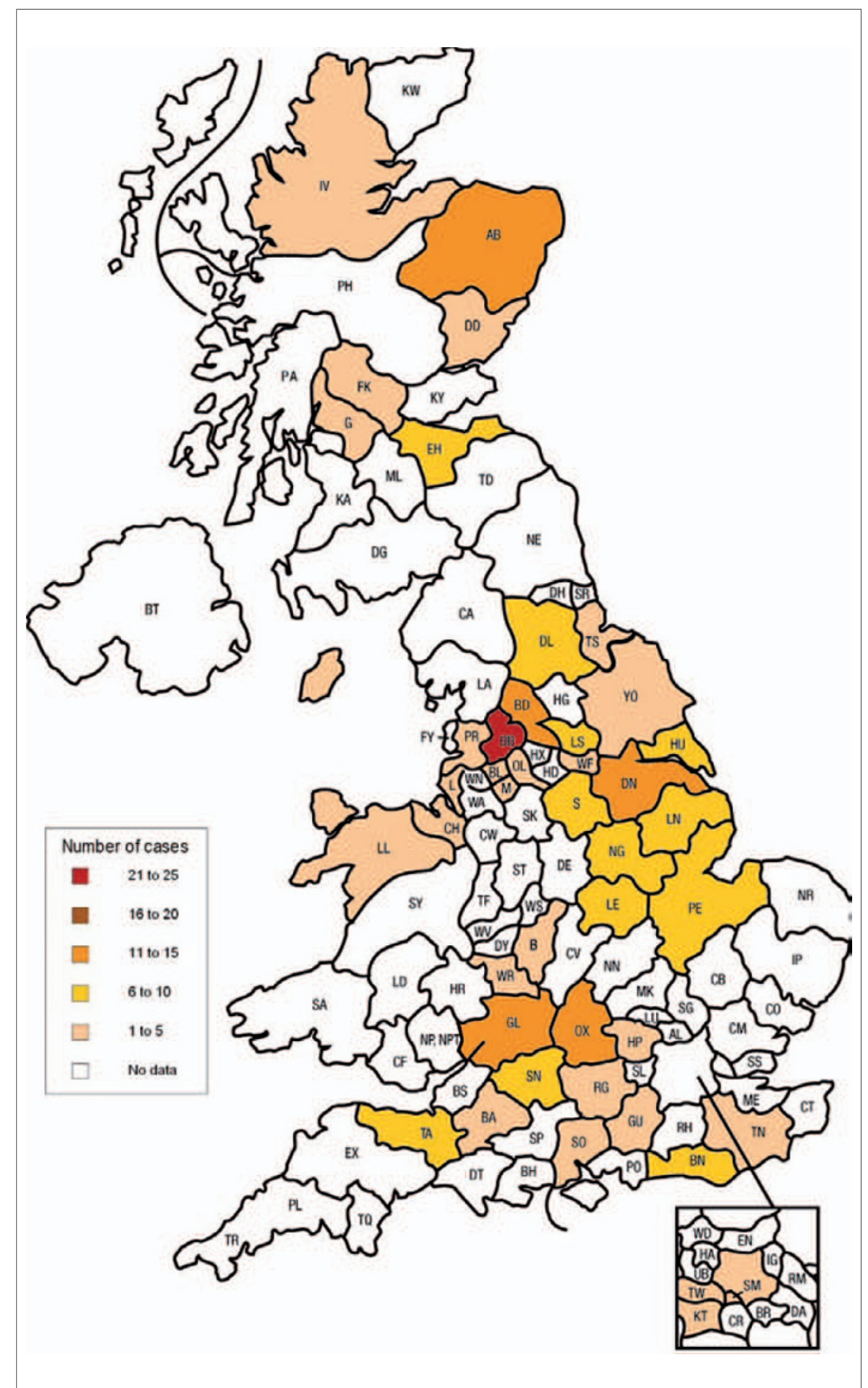

Fig. 3 Number of reported episodes of cervicofacial infection by postcode

$52.6 \%$ sought treatment from primary care dental services yet only 25\% of patients were referred from this source. Fifty-nine percent of patients were referred by accident and emergency or their general medical practitioner. Therefore there must be a proportion of patients who seek treatment from primary care dental services but are ultimately referred by primary care medical services.
Of concern is that $66 \%$ of patients treated by primary care dental services were prescribed oral antibiotics only. This contradicts guidelines from the Faculty of General Dental Practitioners (UK) which state that antimicrobials should be prescribed as an adjunct to removal of the source of infection. ${ }^{10}$ The majority of dentoalveolar infections are caused by necrotic pulp tissue. Removal of the source of infection ie 
the necrotic pulp tissue either by dental extraction or extirpation of the pulp and drainage are well established as the most effective treatment for dentoalveolar infection. ${ }^{11,12}$ The most successful form of drainage is via the soft tissues with drainage via the root canals the next most successful form. ${ }^{13}$ Therefore prescription of oral antibiotics without operative intervention represents an inadequate level of treatment for dentoalveolar infections. However, the number of patients successfully treated by GDPs using oral antibiotics alone is unknown and the number presenting to oral and maxillofacial surgery may be the tip of the iceberg. This warrants further study. Prescription of oral antibiotics can also expose patients to more courses of antibiotics which can lead to development of antibiotic resistance. The incidence of penicillin resistance in odontogenic infection in the UK has

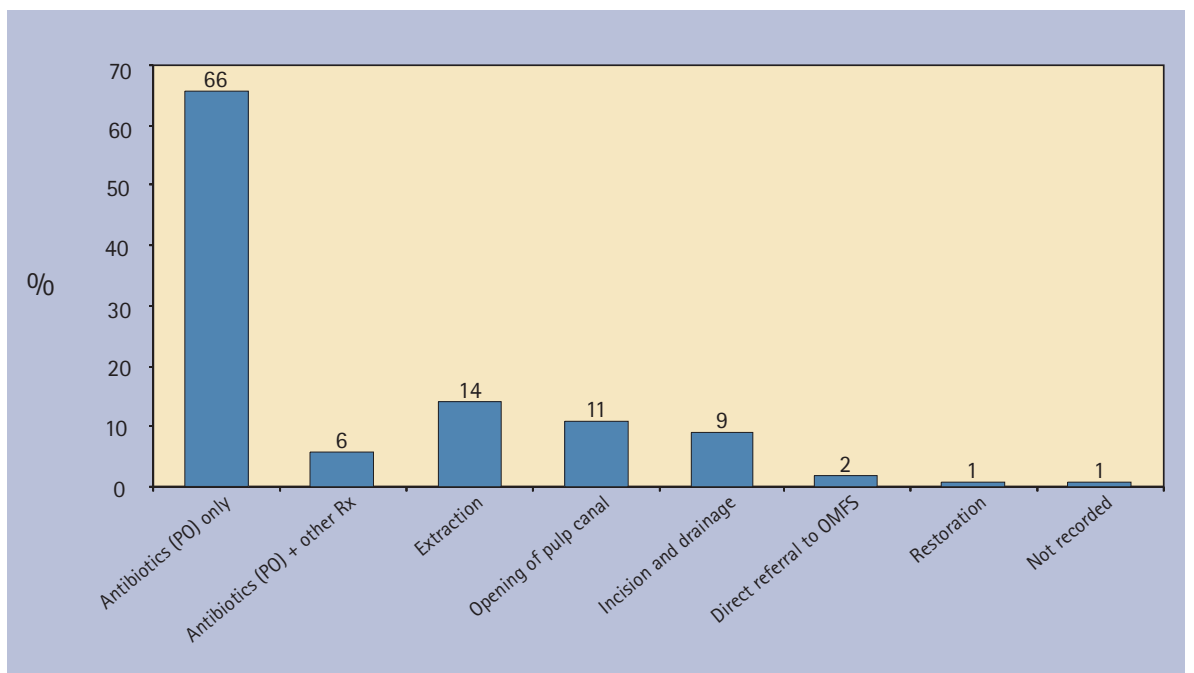

Fig. 4 Treatment provided by primary care dental services

dramatically risen over the last decade and is reported to be at least 55\% now..$^{13,14}$ This has led to the role of penicillin in the treatment of odontogenic infection being questioned and is certainly not as important as achieving drainage. ${ }^{13}$

The proportion of patients receiving operative intervention in dental primary care may improve with education of general dental practitioners or introduction of local or regional audit of the management of dentoalveolar infections. Regional audit has proved successful in rationalising antimicrobial prescribing but will require multidisciplinary co-operation between primary care dental services and oral and maxillofacial surgery. ${ }^{15}$ Financial incentives or introduction of government targets may be the only realistic methods to improve the proportion of patients receiving operative intervention in dental primary care.

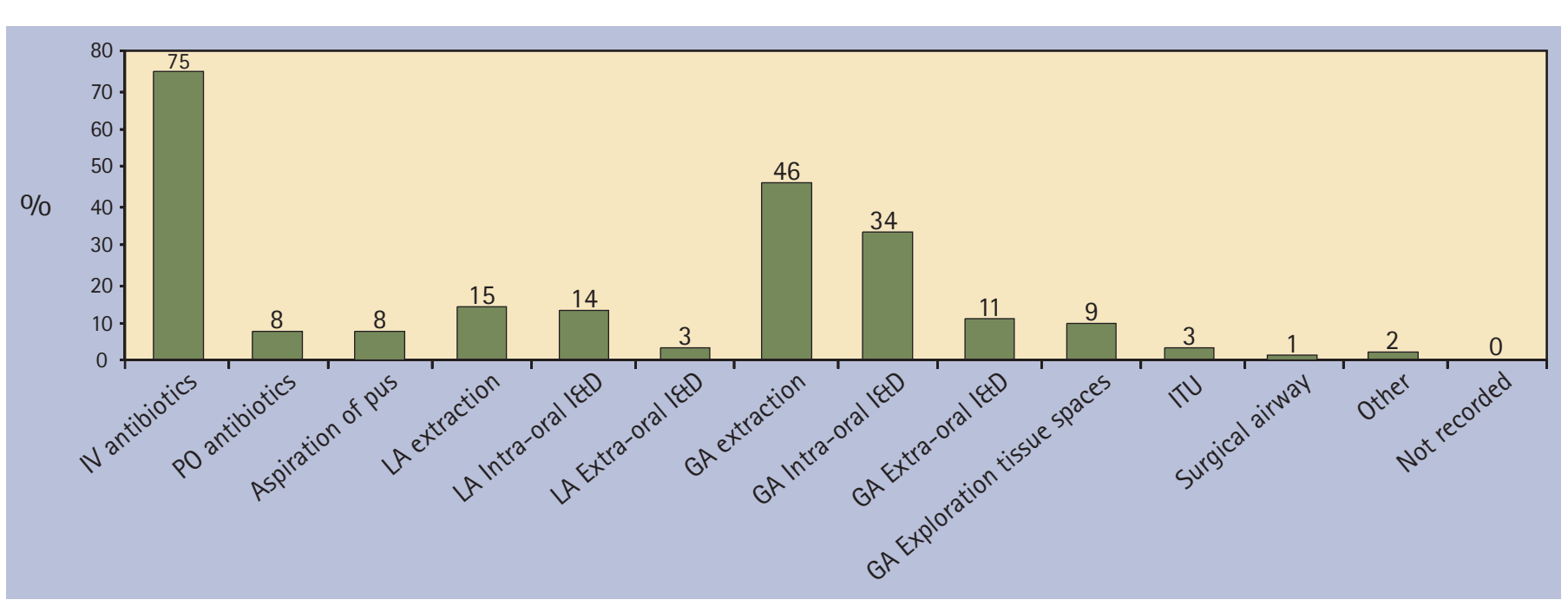

There is significant financial cost and health related morbidity associated with uncontrolled odontogenic infection. Eighty-one percent of patients required hospital admission, 75\% received intravenous antibiotics and 46\% required a surgical procedure under general anaesthesia. Three percent of patients required admission to intensive therapy units and 1\% required a surgical airway. These procedures and inpatient admissions are not only costly in themselves but this unplanned activity can result in cancellation of elective procedures at times of low availability of emergency theatres. In addition the large proportion of patients presenting to accident and emergency increases the accident and emergency workload where staff are not primarily trained to deal with dental problems. These features have a deleterious effect on workforce planning and in provision of elective services in the target driven NHS environment. Although there were no deaths in the audit period, cervicofacial infection of dental origin is still associated with mortality, particularly in patients with complicated medical histories. ${ }^{2,16}$

In summary, by its cross-sectional design, this audit cannot prove an increase in the presentation of cervicofacial infection of dental origin. It does, however, provide a benchmark from which future comparisons can be made. The reasons for presentation of cervicofacial infection to oral and maxillofacial surgery are clearly complex. Lack of access to NHS primary care dental services may be less significant 
than originally thought as only 20 of those who sought dental treatment were unable to access it. A significant proportion of patients preferentially present to primary care medical services rather than dental services. The reasons for this are unclear and further study is indicated. Two thirds of patients treated by primary care dental services received oral antibiotics only which represents an inadequate level of treatment for odontogenic infection.

The authors would like to express their gratitude to BAOMS local and regional audit coordinators in maxillofacial units in the UK who helped and supported information collection.

1. Carter L, Starr D. Alarming increase in dental sepsis. Br Dent J 2006; 200: 11.

2. Cousin G C S, Alam P, Alcock J, Palmer D P et al.
Dying to get to the dentist: fatal oro-facial infections. Br J Oral Maxillofac Surg 2006; 44: 432.

3. Thomas S J, Atkinson C, Hughes C, Revington P, Ness A R. Is there an epidemic of admissions for surgical treatment of dental abscesses in the UK? BMJ 2008; 336: 1219-1220.

4. McMahon J, McCaul J A, Handley T A, Devlin M $F$, Koppel D A. Sepsis syndrome in maxillofacial surgery. Br J Oral Maxillofac Surg 2006; 44: 433.

5. Cousin G C. Potentially fatal oro-facial infections: five cautionary tales. J R Coll Surg Edinb 2002; 47: 585-586.

6. Farrier J N, Kittur M A, Suger A W. Necrotising fasciitis of the submandibular region: a complication of odontogenic origin. Br Dent J 2007; 202: 607-609.

7. Molloy R D, Cousin G C. Biochemical abnormality. Br Dent J 2007; 203: 668 .

8. Greenwood G, Keating J. Fire-fighting disease. [comment] Br Dent J 2006; 200: 27.

9. NHS Dental statistics for England. Quarter 1: 30 June 2006: NHS Information Centre. http://www. ic.nhs.uk/statistics-and-data-collections/primarycare/dentistry/nhs-dental-statistics-for-englandquarter-1:-30-june-2006
10. Adult antimicrobial prescribing in primary care for general dental practitioners. London: Faculty of General Dental Practitioners (UK) The Royal College of Surgeons of England, 2000.

11. Heimdahl A, Nord C E. Treatment of orofacial infections of odontogenic origin. Scand J Infect Dis Supp/ 1985; 46: 101-105.

12. Sandor G K, Low D E, Judd P L, Davidson R J. Antimicrobial treatment options in the management of odontogenic infections. J Can Dent Assoc 1998; 64: 508-514.

13. Kuriyama T, Absi E G, Williams D W, Lewis M A. An outcome audit of the treatment of acute dentoalveolar infection: impact of penicillin resistance. [see comment] Br Dent J 2005; 198: 759-763.

14. Lewis M A O, MacFarlane T W, McGowan D A Antibiotic susceptibilities of bacteria isolated from acute dentoalveolar abscesses. J Antimicrob Chemother 1989; 23: 69-77.

15. Palmer N A, Dailey Y M, Martin M V. Can audit improve antibiotic prescribing in general dental practice? Br Dent J 2001; 191: 253-255.

16. Carter L, Lowis E. Death from overwhelming odontogenic sepsis: a case report. Br Dent J 2007; 203: 241-242. 